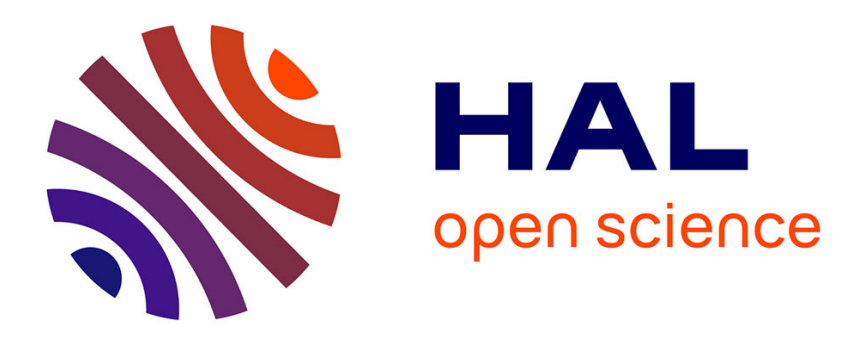

\title{
Capacity evaluation of a quantum-based channel in a biological context
}

\author{
Valeria Loscrì, Anna Maria Vegni
}

\section{To cite this version:}

Valeria Loscrì, Anna Maria Vegni. Capacity evaluation of a quantum-based channel in a biological context. IEEE Transactions on NanoBioscience, 2016, 15 (8), pp.901-907. 10.1109/TNB.2016.2620719 . hal-01386842

\section{HAL Id: hal-01386842 \\ https://inria.hal.science/hal-01386842}

Submitted on 24 Oct 2016

HAL is a multi-disciplinary open access archive for the deposit and dissemination of scientific research documents, whether they are published or not. The documents may come from teaching and research institutions in France or abroad, or from public or private research centers.
L'archive ouverte pluridisciplinaire HAL, est destinée au dépôt et à la diffusion de documents scientifiques de niveau recherche, publiés ou non, émanant des établissements d'enseignement et de recherche français ou étrangers, des laboratoires publics ou privés. 


\title{
Capacity evaluation of a quantum-based channel in a biological context
}

\author{
Valeria Loscrí Member, IEEE, and Anna Maria Vegni Senior Member, IEEE
}

\begin{abstract}
Nanotechnology, as enabler of the miniaturization of devices in a scale ranging from 1 to few hundreds of $\mathrm{nm}$, represents a viable solution for "alternative" communication paradigms that could be effective in complex networked systems, as body area networks. Traditional communication paradigms are not effective in the context of joint body and nano-networked systems, for several reasons, and then novel approaches have been investigated such as nanomechanical, electromagnetic, acoustic, molecular, etc. On the other hand, quantum phenomena represent a natural direction for developing nanotechnology, since it has to be considered as a new scale where new phenomena can occur and can be exploited for information purpose. Specific quantum particles are phonons, the quanta of mechanical vibrations (i.e., acoustic excitations), that can be analyzed as potential information carriers in a body networked context.

In this paper we will focus on the generation of phonons from photon-phonon interaction, by irradiating a sample of human tissue with an electro-magnetic field, and then we will theoretically derive the information capacity and the bit rate in the frequency range $\left[10^{3}-10^{12}\right] \mathbf{H z}$.
\end{abstract}

\section{INTRODUCTION}

Traditional communication paradigms are not suitable in the context of nanotechnology, above all when applied in bio-medical contexts and for body networked systems. Specific features of human body need to be taken into account in order to derive a biological propagation model for nano-communications [1], [2].

Different communication alternatives have been considered, such as molecular and nano-communications [3], acoustic [4], molecular communications based on Forster Resonance Energy Transfer (FRET) phenomenon [5] just to mention some of them. In the last case, authors propose the exploitation of FRET to realize a molecular based physical channel. A step towards the miniaturization at nanoscale level can be also moved by considering quantum phenomena. A very appealing research area involving quantum information science [6], [7] is quantum communication [8], namely the exchange of information encoded in quantum states of matter or quantum bits (known as qubits) between both nearby and distant quantum systems. The key resource behind quantum information science is quantum entanglement, which occurs when the state of a particle interacts with another one and their quantum state cannot be described independently [9].

\footnotetext{
Valeria Loscrí is with INRIA, Lille Nord-Europe, France. Email: valeria.loscri@inria.fr (corresponding author)

Anna Maria Vegni is with Rome TRE University, Department of Engineering, COMLAB Telecommunication lab., Via Vito Volterra, 62 - 00146, Rome, Italy. Email: annamaria.vegni@uniroma3.it

A much shorter version of this work has been accepted to Proc. of ACM NANOCOM 2016, New York City, New York, USA, September 28-30, 2016.
}

A specific type of quantum particles is represented by phonons (or phononics), that represent the quanta of mechanical vibrations or quanta of lattice vibration (i.e. acoustic excitations). In practice, phonons represent quanta of ionic displacement field that describes classical sound waves. Very recently, in the context of fundamental control of phonons, their potentiality in information processing has been shown [10], [11] and due to pressure of Moore's law and Landauer entropy related to the limit of siliconbased electronic computing, there is a great attention devoted to alternative methods of computation and communication. The implementation of a new communication paradigm based on the exploitation of phonons will pave the way to revolutionary applications in the context of communication technologies.

As of today, it is possible to think on the design of devices based on periodic atomic structures, for example realized on planar substrates using litography and plasma etching, engineered for specific wireless communications and sensing applications. Usually, these structures are based on quantum vibrations, namely phonons at megahertz or gigahertz frequencies for their operations and support large phonon band gaps (PnBGs), representing ranges of frequencies that phonons are not allowed to propagate and then can be used to confine and guide the sound particles. Periodic structures have great capabilities in controlling and modifying the flow of particles/waves through the application of the band gap concept and this has been demonstrated through the concept of phonon crystals (PnCs) structures [12]. The feasibility of phonon-routing based information processing in optomechanical crystal circuitry has been recently demonstrated in [13].

In this paper we focus on these specific quantum particles named phonons, that is acoustic excitations that are limited in terms of bandwidth and in terms of ability to transmit information farther than a few millimeter, but can be delayed and stored for significantly longer times than photons counterparts, and can interact resonantly with RFmicrowave electronic systems. On the other hand, quantum information has weird properties that contrast sharply with the familiar properties of classical information. Moreover, quantum phenomena are crucial for the developing of nanotechnology. In fact, nanoscale cannot be considered as another step towards miniaturization, but it represents a kind of new scale [14]. Quantum phenomena can be considered at a purely quantum level or as derived from both quantum and classical laws and components. In this paper, we will focus on a specific context, namely body network systems, where phonons can be generated by photon-phonon interference, by applying an electro-magnetic field on a portion of biological tissue. We will derive the main features of a phonon-based quantum network and, based on the specific representation of this network, we will establish a parallelism with an 
optical quantum network. Phonon-based communication is a method based on a physically existing phenomenon, where phonons (i.e., quantum particles) can potentially provide novel opportunities for quantum communications, as well as nanomedicine applications. Quantum communication networks are expected to provide high speed communications than "traditional" counterparts. Finally, phonon-based communications can represent a viable technique also in the context of nanomedicine applications, as an alternative to well-known molecular communications [15].

Leveraging on these assumptions, we will characterize phonons as information carriers, and will derive a characterization of the information capacity from a theoretical point of view. Furthermore, since we can assume that the biological information processing takes place at the challenging regime where quantum meets classical physics, we will focus on a specific biological scenario with a sample of human tissue irradiated with an electromagnetic field, representing the source generation of a photon-phonon interaction. Notice that the overall communication system is only based on the phonons. The interaction between photons and phonons allows the phonons generation. Then, in this paper, we derive the phonon-based capacity. Then, we will derive the theoretical information capacity and the bit rate of this system. The formal derivation of the information capacity is based on the concept that the initial representation of the phononbased quantum network is formally equivalent to an optical quantum networks, but in contrast to high frequency optical fields, there is a generation of heat phonons, called thermal population, that is not negligible. We will characterize this thermal population in the body system context.

The rest of the paper is organized as follow. In Section II, we explain the system model as represented in our paper. In Section III we derive a theoretical evaluation of the capacity and the bit rate. In Section IV we show a concrete example of biological tissue irradiated with an electromagnetic field and we show the capacity information and the bit rate in the specific case under analysis. Finally, conclusions and future directions are drawn at the end of the paper.

\section{Phonon Communication System}

In analogy with [16], our phonon-based quantum communication system is composed by $N$ elementary components that we refer as nodes, and the phonon channel. The connection among the nodes is established through a quantum bus, and the $i$-th node is associated with a two-level system, namely the qubit (quantum bit) with the information bit encoded as $\mid 0>$ and $\mid 1>$, as depicted in Fig. 1. Each node is represented by a cavity (element), where quantum information is encoded in states $\mid 0>$ and $\mid 1>$. We assume that node $i$ is a transmitter, while node $j$ a receiver. The qubits interact with the phonon cavities, also weakly coupled to the quantum bus. The qubit propagating in the quantum bus are depicted as grey rectangles, while the qubit transmitted by node $i$ or received by node $j$ are represented by black rectangles. The interaction from a cavity to the next one generates a phonon, and it is shown as a propagating wave.

Notice that the quantum information is transmitted through propagating phonons. This aspect distinguishes the proposed approach from the Nanoscale Heat Communications (NHC) [17], where the information is encoded in tem-

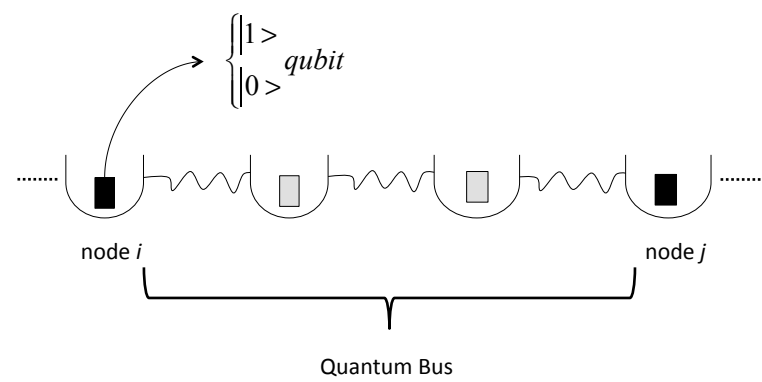

Fig. 1. A generic phonon-based network, where information is encoded in states $\mid 1>$ and $\mid 0>$

perature signals by using Magneto-Caloric Effects (MCE). This implies the use of magnetic materials exposed to a varying magnetic field.

The communication system can be described through an Hamiltonian $H$. The Hamiltonian operator $H$ describes the energy of the system, and as any other quantum system, the state of a qubit will evolve under the influence of its Hamiltonian.

The operator $H$ is composed by three fundamental components, that include the energy related to $(i)$ the nodes, (ii) the channel, and (iii) the interaction between nodes and channel, i.e.

$$
H=\sum_{i=1}^{N} H_{\text {node }}^{i}+H_{\text {channel }}+H_{\text {int }} .
$$

In (1) the operator $H_{\text {node }}$ related to the $i$-th node can be expressed through a temporal dependency as:

$$
H_{\text {node }}^{i}(t)=\omega_{m} b_{i}^{\dagger} b_{i}+\frac{\triangle_{q}^{i}(t)}{2} \sigma_{z}^{i}+\lambda_{i}(t)\left(\sigma_{+}^{i} b_{i}+\sigma_{-}^{i} b_{i}^{\dagger}\right),
$$

where the Pauli operators are as follows i.e.,

$$
\sigma_{+}=\left[\begin{array}{ll}
0 & 1 \\
0 & 0
\end{array}\right], \sigma_{-}=\left[\begin{array}{ll}
0 & 0 \\
1 & 0
\end{array}\right], \sigma_{z}=\left[\begin{array}{cc}
1 & 0 \\
0 & -1
\end{array}\right] .
$$

Moreover, in (2), $b_{i}$ represents the bosonic operators, $\omega_{m}$ is the phonon mode frequency, $\triangle_{q}^{i}(t)$ is the qubit frequency splitting, $\lambda_{i}(t)$ is the qubit-resonator coupling, and the term $\lambda_{i}(t)\left(\sigma_{+}^{i} b_{i}+\sigma_{-}^{i} b_{i}^{\dagger}\right)$ characterizes the coupling between the qubits and the local modes. The interactions of a qubit and the resonators, as described in the above equation, have been characterized in literature [16].

In the phonon-based quantum network, the nodes communicate by means of a quantum channel as represented in Fig. 1, where we show a representation of a generic phononbased network. Specifically, nodes are connected through a quantum channel (quantum bus) constituted by a chain of $N_{C h}$ coupled mechanical resonators, expressed as:

$$
H_{\text {channel }}=\sum_{l=1}^{N_{C h}} \frac{p_{l}^{2}}{2 m}+\frac{1}{2} m \omega_{0}^{2} x_{l}^{2}+\frac{k}{2} \sum_{l=1}^{N_{C h}-1}\left(x_{l}-x_{l+1}\right)^{2},
$$

where $x_{l}$ is the position operator, $m$ is the effective mass, $p_{l}$ is the momentum operator, $\omega_{0}$ is the bare oscillating frequency, and $k$ is a spring constant that takes into account the nearest neighbor coupling. In the case of $N_{C h} \gg 1$, that represents the scenario we are interested to as we assume 
extended arrays, the mechanical quantum channel $H_{\text {channel }}$ can be represented by a set of plane wave modes i.e.,

$$
H_{\text {channel }}=\sum_{q} \omega_{q} b_{q}^{\dagger} b_{q},
$$

where $\omega_{q}$ is the phonon dispersion relation, and

$$
\left[b_{q}, b_{q}^{\dagger}\right]=\delta_{q, q^{\prime}},
$$

with [ ] as the canonical commutation relation, and $\delta$ as the Kronecker delta. If we consider a lattice space $a$, with $a \backsim 1 \mu \mathrm{m}$, the momentum label $q$ is restricted to the first Brillouin zone $q \in\left(\frac{\pi}{a}, \frac{\pi}{a}\right]$. Moreover, by assuming a phononic band gap structure as realized in [18], a resonator $l$ corresponds to a vibrational mode of a unit cell. It follows that it is possible to identify a frequency range $\Delta \omega$ (away from the band edges), where the resonator exhibits an approximately linear dispersion i.e., $\omega_{q} \approx \tilde{\omega}_{0}+c|q|$, where $\tilde{\omega}_{0}$ is frequency offset, and $c$ is the speed of sound.

We can derive the normalized displacement field as

$$
\Phi(z)=\Phi_{R}(z)+\Phi_{L}(z),
$$

where the two components $\Phi_{R}(z)$ and $\Phi_{L}(z)$ represent right and left moving mechanical excitations of the phononic channel, respectively, i.e.

$$
\begin{gathered}
\Phi_{R}(z)=\sqrt{\frac{2 c}{L}} \sum_{q>0} e^{i q z} b_{q}, \\
\Phi_{L}(z)=\sqrt{\frac{2 c}{L}} \sum_{q>0} e^{-i q z} b_{-q},
\end{gathered}
$$

where $L$ is the waveguide length, and $c$ is the speed of light. Notice that the waveguide represents the phonon channel, namely the sequence of nodes (or cavities) where there is the qubit interacting with the phonon cavities.

If we assume that the frequency of the local modes $\omega_{m}$ lies within this frequency range, $\Delta \omega$, then the local resonators and the waveguide modes coupling are represented through:

$$
H_{\text {int }} \approx \sqrt{\frac{\gamma}{2}} \int_{0}^{L} d z \sum_{i=1}^{N} \delta\left(z-z_{i}\right)\left(b_{i}^{\dagger} \Phi(z)+b_{i} \Phi^{\dagger}(z)\right),
$$

where $z_{i}$ represents the position of the nodes along the waveguide, and $\gamma$ is the total decay rate of the local resonator modes into the waveguide.

Moreover, if we assume that there is a linear dispersion, the field obeys to the following expressions i.e.,

$$
\begin{gathered}
{\left[\Phi_{R}(z, t), \Phi_{R / L}^{\dagger}\left(z^{\prime}, t^{\prime}\right)\right]=0} \\
{\left[\Phi_{R / L}(z, t), \Phi_{L}^{\dagger}\left(z^{\prime}, t^{\prime}\right)\right] \approx \exp ^{-j \tilde{\omega}\left(t-t^{\prime}\right)} \delta\left(t-t^{\prime} \mp\left(z-z^{\prime}\right) / c\right) .}
\end{gathered}
$$

In realistic cases, we need to take into consideration also phonon losses, backscattering, and re-thermalization effect. Right now, we have characterized the quantum phononic network from an energy point of view, by considering the Hamiltonian operator. The network derived is formally equivalent to an optical quantum network and then, it is possible to use it in a similar fashion to transfer quantum states among the nodes. However, there are differences that we need to take into consideration, such as that at high frequencies, the heat bath population of phonons is normally not negligible. This implies that if transfer state protocols as conceived in [16] are considered, specific filters have to be assumed in the case of phonon communications. This kind of protocols are supposed to be developed in periodic structures, based on the exploitation of band gap concept.

On the other hand, the context under analysis in this paper is related to body networked systems where we cannot consider periodic structures, and also the generation of thermal bath phonons is an interesting subject to be investigated as potential carriers of information. Based on these considerations, in the next section we will derive the theoretical capacity of a quantum based system, formally represented as our system.

\section{THEORETICAL INFORMATION CAPACITY AND BIT RATE}

In this paper we are interested to derive a theoretical information capacity suitable for the phonon channel model, as presented in Section II. For this aim, we start by considering the computation of the information capacity in a "traditional case". In a classical communication channel, the information capacity, namely the maximum number of bits that can be delivered in a reliable way, is given by considering the maximum of the Von Neuman entropy [19], [20] as:

$$
S(\rho)=-\operatorname{Tr}\left[\rho \log _{2} \rho\right],
$$

where $\operatorname{Tr}[]$ is the matrix trace operator, and $\rho$ represents the input states. If we assume that the entropy $S(\rho)$ is maximized on the states where the average energy is $E=\operatorname{Tr}[\rho H]$, and $H$ is the Hamiltonian, then we can solve the constrained maximization of $S(\rho)$ by using standard variational methods. This implies to solve the following equation:

$$
\delta\left\{S(\rho)-\frac{\beta}{\ln 2} \operatorname{Tr}[H \rho]-\frac{\beta^{\prime}}{\ln 2} \operatorname{Tr}[\rho]\right\}=0,
$$

where $\delta$ is the Dirac function, $\beta$ and $\beta^{\prime}$ are Lagrange multipliers that consider the normalization constraint $\operatorname{Tr}[\rho]=1$, and the $\ln 2$ factor is introduced to make possible the use of natural logarithms. Equation (13) can be solved by density matrix as

$$
\rho=\frac{e^{-\beta H}}{Z(\beta)},
$$

where $Z(\beta)=\operatorname{Tr}\left[e^{-\beta H}\right]$ is the partition function of the system, and is determined by solving

$$
E=-\frac{\partial}{\partial \beta} \ln [Z(\beta)]
$$

It follows that from (12) and (14), we can easy compute the capacity of the quantum channel as:

$$
C=S\left[\frac{e^{-\beta H}}{Z(\beta)}\right]=\frac{\beta E+\ln [Z(\beta)]}{\ln 2} .
$$

Of course, this represents an ideal capacity, since for real systems we must also account for intrinsic phonon losses, backscattering and re-thermalization effect.

From (2) we can argue that the evaluation of the system capacity can be done through the evaluation of its partition function $Z(\beta)$. Generally, the expression of $Z(\beta)$ is not easy 
to be derived. In the case of non-interacting bosonic systems, as the case under analysis in this paper, we have seen that the Hamiltonian of channel is given by (4) where we do not consider $\hbar$ since we have supposed to be in the case where $\hbar=1$. In this case, the partition function can be rewritten as:

$$
Z(\beta)=\prod_{q} \sum_{n_{q}=0}^{\infty} e^{-\beta \omega_{q} n_{q}}=\prod_{q} \frac{1}{1-e^{-\beta \omega_{q}}}
$$

This means that the associate capacity will be determined by the spectrum $\omega_{q}$ of the system. If we consider the case of narrowband systems, namely only a mode of frequency $\omega$ exists, the related capacity $C_{n b}$ will be given by:

$$
C_{n b}=g\left(\frac{E}{\hbar \omega}\right)
$$

where $\hbar=1$, and $g(\cdot)$ is a function expressed as

$$
g(x)=(1+x) \log _{2}(1+x)-x \log _{2}(x),
$$

with the condition that $g(0)=0$.

Since we are considering the case of $N_{C h} \gg 1, H_{\text {channel }}$ can be represented as a dense set of plane wave modes, and then we consider an infinite collection of equi-spaced frequencies i.e., $\omega_{q}=q \delta \omega$, employed for $q \in H_{\text {channel }}$. The equation of the capacity for wideband systems will be then:

$$
C_{w b} \simeq \frac{\pi}{\ln 2} \sqrt{\frac{2 E}{3 \hbar \delta \omega}}
$$

which is valid if $\hbar \delta \omega \ll E$, that will become $\delta \omega \ll E$ in our case. For the interested reader, in Appendix A we report the derivation of the capacity expression.

Finally, by assuming the power $P$ as energy transmitted per time unit i.e.,

$$
P=\frac{\delta \omega E}{2 \pi}
$$

it follows that we can express the rate $R$ as qubits transmitted per time unit, as

$$
R=\frac{1}{\ln 2} \sqrt{\frac{\pi P}{3 \hbar}}
$$

This formula represents the ideal case where phonons persist indefinitely. In practice, it does not take into consideration that phonons have a limited lifetime that we will indicate as $\tau$ [s]. By taking into account the phonon lifetime (i.e., $\tau$ ) as a first order approximation, we can multiply the energy of the phonons by $\tau / T_{s}$, where $T_{s}$ represents the periodic time for the streaming of photons is represented as:

$$
T_{s}=\frac{1}{n_{\text {photons }}},
$$

where $n_{\text {photons }}$ is the number of photons incident on one cell. Then, the rate with the phonon lifetime dependency i.e., $R(\tau)$, can be expressed as:

$$
R(\tau)=\frac{1}{\ln 2} \sqrt{\frac{\pi P\left(\tau / T_{s}\right)}{3 \hbar}} .
$$

\section{CAPACITY OF PHONON-BASED QUANTUM NETWORK}

Based on the results obtained in the previous section, we will examine the capacity of a phonon-based quantum channel for biomedical applications, assuming an electromagnetic radiation is incident to a portion of human tissue. Notice that for this specific context, the possible side effects are exactly the same when a mobile phone is used.

Let us consider the photon-phonon interaction as mediated by a homogeneous sample of identical elements (namely, the human cells), which are in harmonic modes of vibration. We can consider a system of $N$ vibrating elements, each of them has an energy as:

$$
E_{n}=\left(N+\frac{1}{2}\right) h f
$$

where $E_{n}$ represents the energy of the $n$-th stationary state, $f$ is the frequency of a normal mode that an element vibrates accordingly to, and $h$ is the Planck's constant equal to $6.62606957 \times 10^{-34}[\mathrm{~J} \mathrm{~s}]$.

When an element absorbs totally or partially a photon with an initial frequency $f_{i n}$, there is a phonon production that is regulated by Raman-Stokes scattering effect [21], and an excited phonon will have a frequency $f$. This results in the $n$-th element to acquire a higher energy, and the absorbed energy will be

$$
E_{n}-E_{0}=n_{p} h f,
$$

where $n_{p}$ represents the number of phonons. It follows that the scattered photon will end up with energy $h f_{s}$, and so we have

$$
h f_{\text {in }}=h f_{s}+n_{p} h f .
$$

Notice that each element ${ }^{1}$ cannot absorb photon that may elevate its energy to a state higher than $k_{B} T$ that is the thermal noise, i.e.

$$
E_{n}=\left(N+\frac{1}{2}\right) h f \leq k_{B} T,
$$

where $k_{B}=8.6173324 \times 10^{-5}\left[\mathrm{eVK}^{-1}\right]$ is the Boltzmann constant. This means that the number of phonons depend on both the frequency and the temperature as:

$$
0 \leq n_{p} \leq \frac{k_{B} T}{h f}-\frac{1}{2} .
$$

In order to simplify the treatment of the problem, we make some assumptions that we will be able to relax in future works. Specifically, we assume that the flux of photons scattered on the basis of Raman-Stokes laws is generated in such a way that the number of photons is equal to the number of elements. The number of elements (i.e., $N$ ) will coincide with the number of cells and we will assume that this will be in the order of $\simeq 10^{12}$. This value comes out from the specific context considered.

In fact, we assume that the phonons and the photons incident on the biological sample are the result of the electromagnetic waves emitted from an electromagnetic source (e.g. a common cellphone). We assume a power incident on $1 \mathrm{~cm}^{3}$ on the human tissue. Normally, human cells are of the order of $1 \mu \mathrm{m}$ far from each other and the number of cells in $1 \mathrm{cc}$ will be in the order of $10^{12}$.

\footnotetext{
${ }^{1}$ We assume an element coincides with a biological cell.
} 


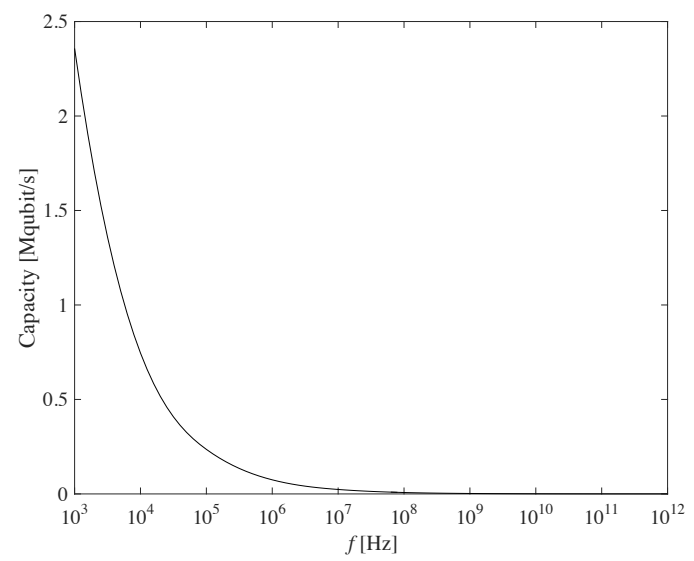

Fig. 2. Capacity of phonon-based quantum channel versus frequency.

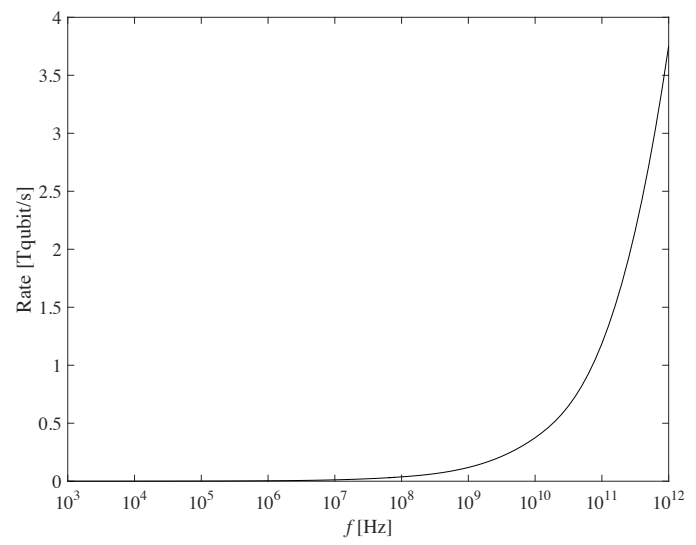

Fig. 3. Rate of phonon-based quantum channel versus frequency.

In such a scenario, when a photon interacts inelastically with one element (i.e. one cell), it contributes to generate a random number of phonons with a randomized value of frequency. Notice that we are assuming the maximum number of phonons generated since we do not have prior information regarding the real number of phonons that can be created in a single collision between a photon and a cell. Furthermore, we assume that all the phonons will have the same frequency $f$. This means that the number of phonons in (29) will be exactly equal to the upper bound i.e., $\frac{k_{B} T}{h f}-\frac{1}{2}$. Then, it follows that the capacity in (20) will become:

$$
C_{w b} \simeq \frac{\pi}{\ln 2} \sqrt{\frac{2 n+1}{3 \delta}} .
$$

In practice, we have determined that the capacity in the case of a homogeneous wideband channel is proportional to the number of phonons that are inversely proportional to the frequency. In the specific case of a biological tissue, there exists a frequency band in the range $\left[10^{3}, 10^{12}\right] \mathrm{Hz}$, where the energy absorbed reaches its maximum value and it seems that there is no appreciable absorption beyond this band. So, with the aim of characterizing the phonon-based quantum channel capacity, we have to limit to this specific frequency range.

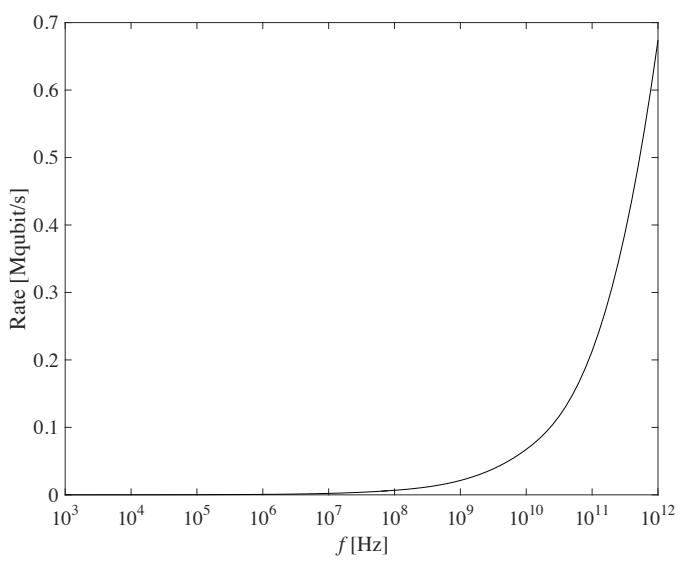

Fig. 4. Rate of phonon-based quantum channel versus frequency, including the phonon lifetime $\tau$.

\section{A. Performance Evaluation}

In this section, we will evaluate the information capacity in the case of a sample of biological tissue at a typical temperature of $37^{\circ} \mathrm{C}$, where the thermal noise $k_{B} T \simeq 27 \mathrm{meV}$ with the values of emitted power as described above.

In Fig. 2 we show the capacity of the system by varying the frequency in the range $\left[10^{3}, 10^{12}\right] \mathrm{Hz}$. As expected, since the number of phonons, at a fixed temperature, is higher at lower frequency modes, the capacity will be higher at lower frequencies. It is worth to recall that we are considering a $1-t o-1$ correspondence in the phonon generation from one incident photon. This means that for each photon generated there will be one phonon.

In a more realistic condition, we should take into account that the photons are inelastically scattered and a ratio of around $10^{-4 \%}$ is engaged in phonon generation. Since this factor impacts on the number of phonons, based on the model we have derived, it will also impact on the energy and then on the total capacity. On the other hand, we did not make any assumptions on the exposure time i.e., the time interval of irradiation on the biological sample from an incident electromagnetic wave, for the sake of simplicity of the analysis. But, it is obvious that as the time of exposure to the electromagnetic field increases, the number of incident photons will increase as well.

In Fig. 3 we show the rate $R$ (as bits transmitted per unit time) in an ideal case (i.e. phonons are considered persistent over the time) as computed in (22), by considering the power $P$ as the amount of energy transmitted per unit time. In this specific case, we have considered a transmission time equal to $2 \pi /(\delta \omega)$. Differently from Fig. 2 where higher capacity is experienced for lower frequencies, the rate increases for higher frequencies i.e., $>1 \mathrm{GHz}$, due to the frequency dependency of the transmission time. In fact, the higher is the frequency, the lower will be the transmission time and consequently the rate. For this reason, we can observe an opposite trend in the capacity and rate shapes. In Fig. 3, maximum value (i.e., 3.75 Tqubit/s) is obtained for the frequency upper bound of $1 \mathrm{THz}$.

Notice that Fig. 3 represents the rate by assuming that the phonons remain in the excited state without including their lifetime. In Fig. 4 we depict $R(\tau)$ as computed in (24). It is worth to notice that in this specific case we assumed 
that the number of photons incident on one cell is 1 , that means that there is a correspondence $1-t o-1$ between the number of cells and the number of photons. As expected, we observe a reduction of rate to the order of [Mqubit/s], still for frequency values $>1 \mathrm{GHz}$. Also in this case, maximum value (i.e., 0.673 Mqubit/s) is reached for $1 \mathrm{THz}$.

\section{CONCLUSIONS}

In this paper we have investigated quantum mechanics as a viable way to meet the nanotechnology. In particular, we analyzed a specific quantum particle, named phonon (or phononics), representing acoustic excitations, from a communication perspective. Specifically, we assumed that these quantum particles can be used to encode the information, namely phonons are considered as potential information carriers. Normally, these particles are studied in periodic structures, as crystals structures, that provide a promising platform for realizing phonon networks in practice.

In this work, we have analyzed a different context, represented by a sample of biological tissue irradiated with an electromagnetic source that generates a photon-phonon interaction. The phonons generated in this way represent the so-called "thermal bath phonon modes". Following the concept of photon-phonon interaction, it is possible to derive the theoretical information capacity and the rate of these generated phonons. In practice, we have analyzed their communication capability through a simple model that has as objective to show the feasibility of a nano-communication paradigm in body networked systems based on phonon particles.

The work dealt in this paper has as objective to start the characterization of a phonon-based networked system, that could be exploited in a future for addressing the design of nano-motors with specific features matching the environment under analysis. As future investigations, we aim to refine the model presented by taking into account of real phenomena and their impact on the Hamiltonian that represents the system. We will consider non-linear terms in the Hamiltonian system that takes into consideration interactions between particles and the propagation medium. Finally, as future work, it will be interesting to investigate the impact of different tissues on phonon-based communications. Indeed, the generation of phonons strictly depends on the inherent structure of the specific biological material.

\section{APPENDIX}

\section{A. Theoretical derivation of the expression of the quantum} capacity (18)-(20)

The possible amount of information transferred from a source to a receiver via a quantum system is bounded through the quantum entropy function. The capacity in a narrowband system is computed as:

$$
C=g\left(\frac{E}{\hbar \omega}\right),
$$

and derives from the characterization of the capacity of a lossy bosonic channel (in bits per channel use) as

$$
C=\max _{N_{k}} \sum_{k} g\left(\eta_{k} N_{k}\right)
$$

where $N_{k}$ is the modal average phonon-number sets $\left\{N_{k}\right\}$ that must satisfy the following energy constraint

$$
\sum_{k} \hbar \omega_{k} N_{k}=E
$$

with $\omega_{k}$ as the frequency of the $k$-th mode, and $\eta_{k}$ as the transitivity (quantum efficiency).

In (32), $g(x)$ is the Shannon entropy of the Bose-Einstein distribution with mean $x$, as demonstrated for the first time in [22]. In the expression of $g(x)$ in (19), the base used for the logarithm sets the units for measuring information content, i.e. the base $e$ leads to nats, while the base 2 leads to bits.

The maximization is performed on the modal average phonon-number sets $\left\{N_{k}\right\}$ that satisfy the energy constraint (33). The capacity expression (31) can be simplified by using standard variational techniques, since the maximization is constrained and then we can rewrite it as:

$$
C=\sum_{k} g\left(\eta_{k} N_{k}(\beta)\right),
$$

where $N_{k}$ represents the optimal phonon-number distribution, and $\beta$ is a Lagrange multiplier determined through the constrained energy transmitted. In the case of narrowband channel, there is a single mode of frequency $\omega$ employed. In this specific case, (34) becomes:

$$
C=g\left(\frac{\eta E}{\hbar \omega}\right),
$$

since we have considered the average phonon number i.e., $N=\frac{E}{\hbar \omega}$, as input.

In the case of a wideband channel with uniform transmittivity i.e., $\eta_{k}=\eta$, where there is a set of equi-spaced frequencies i.e., $\omega_{k}=k \delta \omega$ (with $k \in \mathbb{N}$ ), we can replace the sums in (33) and (34) with integrals and we obtain that the power constraint becomes:

$$
P=\int_{W} \frac{\hbar \omega / \eta_{k}}{e^{\hbar \omega / \eta_{k}}-1} d \omega,
$$

where $W$ is the frequency band (modes per second). Then, the capacity can be rewritten as:

$$
C=\int_{W} g\left(\frac{1}{e^{\hbar \omega / \eta_{k}}-1}\right) d \omega .
$$

By solving (36) and (37), we obtain:

$$
C=\frac{\sqrt{\eta}}{\ln 2} \sqrt{\frac{\pi P}{3 \hbar}} T,
$$

where $T=\frac{2 \pi}{\delta \omega}$.

Finally, if we consider the lossless case (i.e., $\eta=1$ ) [22], the capacity becomes:

$$
C=\frac{\pi}{\ln 2} \sqrt{\frac{2 E}{3 \hbar \delta \omega}} .
$$

This result has been obtained by showing that (39) is simultaneously a lower bound and an upper bound for the capacity [23].

\section{REFERENCES}

[1] Q. H. Abbasi, H. El Sallabi, N. Chopra, K. Yang, K. Qaraqe, and A. Alomainy. Terahertz channel characterisation inside the human skin for nano-scale body-centric networks. IEEE Transactions on THz Science and Technology, 2016. 
[2] K. Yang, N. Chopra, M. Munoz, Q. H. Abbasi, Y. Hao, and A. Alomainy. Effects of non-flat interfaces in human skin tissues on the in-vivo tera-hertz communication channel. Elsevier Nano Communication Networks, November 2015.

[3] I. F. Akyildiz, J.M. Jornet, and M. Pierobon. Nanonetworks: A new frontier in communications. Comm. of the ACM, 54(11):84-89, 2011.

[4] V. Loscrí and A.M. Vegni. An acoustic communication technique of nanorobot swarms for nanomedicine applications. IEEE Trans. on Nanobioscience, 14(6):598-607, 2015.

[5] M. Kuscu and O.B. Akan. Coverage and throughput analysis for fretbased mobile molecular sensor/actor nanonetworks. Elsevier Nano Commmunication Networks, 5:45-53, 2014.

[6] J. Stajic. The future of quantum information processing. Science, 339(6124):1163, Mars 2013.

[7] JA. M. Eltony, D. Gangloff, M. Shi, V. Vuletic A. Bylinskii, and I. L. Chuang. Technologies for trapped-ion quantum information systems - progress towards scalability with hybrid systems. Quantum Physics, pages 1-19, Mars 2016.

[8] H. J. Kimble. The quantum internet. Nature, 453:1023-1030, June 2008.

[9] L.M. Duan, M. D. Lukin, J. I. Cirac, and P. Zoller. Long-distance quantum communication with atomic ensembles and linear optics. Nature, 414:413-418, November 2001.

[10] S. R. Sklan. Splash, pop, sizzle: Information processing with phononic computing. AIP Advances, 5(053302):1-29, November 2001.

[11] L. Wang, G. Zhang, G. Wu, N. Yang, and B. Li. Phononics: A new science and technology of controlling heat flow and processing information by phonons. 2010 .

[12] M.M. Sigalas and E.N.Economou. Elastic and acoustic wave bandstructure. Journal of Sound and Vibration, 158(2):377-382, 1992.

[13] K. Fang, M. H. Matheny, X. Luan, and O. Painter. Phonon routing in integrated optomechanical cavity-waveguide systems. PhysicsOptics, pages 1-16, August 2015.

[14] I.F. Akyildiz, J.M Jornet, S. Balasubramanian, and Y. Koucheryavy. Internet of bionanothings. IEEE Comm. Mag., 53(3):32-40, 2015.

[15] L. Felicetti, M. Femminella, G. Reali, and P. Li. Applications of molecular communications to medicine: A survey. Nano Communication Networks, 7:27 - 45, 2016.

[16] S.J. Habraken, K. Stannigel, M.D. Lukin, P. Zoller, and P. Rabl. Continuous mode cooling and phnon routers for phononic quantum networks. New Journal of Physics, 14:1-33, November 2012.

[17] D. Kilinc and O. B. Akan. A theoretical modeling and analysis of communication via heat flow at nanoscale. IEEE Transactions on Communications, 62(10):3600-3609, Oct 2014.

[18] R. H. Olsson and I. El-Kady. Microfabricated phononinc crystal devices and applications. IOPscience Measurement Science and Technology, 20(1):1-13, November 2008.

[19] V. Giovannetti, S. Lloyd, and L. Maccone. Capacity of nonlinear bosonic systems. Physical Review A, 70:012307-1-012307-6, 2004.

[20] Alexander S. Holevo. Bounds for the quantity of information transmitted by a quantum communication channel. Problems of Information Transmission, 9:177-183, 1973.

[21] C.V. Raman and K. S. Krishnan. The raman effect - inelastic insight. Nature, 121, 1928.

[22] H.P. Yuen and M. Ozawa. Ultimate information carrying limit of quantum systems. Phy. Rev. Lett., 70, January 1993.

[23] V. Giovannetti, S. Guha, S. Lloyd, L. Maccone, J.H. Shapiro, and H.P. Yuen. Classical capacity of the lossy bosonic channel: The exact solution. Phy. Rev. Lett., 92, January 2004. 